\title{
DENSITY OF THE POLYNOMIALS IN THE HARDY SPACE OF CERTAIN SLIT DOMAINS
}

\author{
JOHN AKEROYD
}

(Communicated by Paul S. Muhly)

\begin{abstract}
In this article we construct a Jordan arc $\Gamma$ in the complex plane, with endpoints 0 and 1 , such that the polynomials are dense in the Hardy space $H^{2}(\mathbb{D} \backslash \Gamma) ; \mathbb{D}:=\{z \in \mathbb{C}:|z|<1\}$.
\end{abstract}

It is well known that if $G=\{z \in \mathbb{C}:|z|<1\} \backslash[0,1] \quad(\mathbb{C}$ denotes the complex plane), then the polynomials are not dense in the Hardy space $H^{2}(G)$. One of the assertions of this paper, however, is that there are regions $D$ of the same sort as $G$ such that the polynomials are dense in $H^{2}(D)$. In fact, we construct a homeomorphic image $\Gamma$ of the interval $[0,1]$, where $\Gamma$ has endpoints 0 and 1 , and $\Gamma \backslash\{1\} \subseteq \mathbb{D}:=\{z \in \mathbb{C}:|z|<1\}$, such that the polynomials are dense in $H^{2}(\mathbb{D} \backslash \Gamma)$.

Recall that if $D$ is a bounded Dirichlet region, then the Hardy space $H^{2}(D)$ is the collection of functions $f$ that are analytic in $D$ such that $|f|^{2}$ has a harmonic majorant on $D$. Furthermore, for any point $z_{0}$ in $D$ (norming point), the mapping $\|\cdot\|_{z_{0}}: H^{2}(D) \rightarrow \mathbb{R}$ defined by $\|f\|_{z_{0}}=\left(u_{f}\left(z_{0}\right)\right)^{1 / 2}$, where $u_{f}$ is the least harmonic majorant of $|f|^{2}$ on $D$, is a norm on $H^{2}(D)$, and, under this norm, $H^{2}(D)$ forms a Banach space (cf. [6]). By Harnack's inequality, different norming points yield equivalent norms. We let $\omega\left(\cdot, D, z_{0}\right)$ denote harmonic measure on $\partial D$ evaluated at $z_{0}$. Notice that if $f$ is analytic on $D$ and continuous on $\bar{D}$, then $f \in H^{2}(D)$ and

$$
\|f\|_{z_{0}}=\left\{\int|f(\zeta)|^{2} d \omega\left(\zeta, D, z_{0}\right)\right\}^{1 / 2} .
$$

1. Definition. A function $\gamma:[0,1] \rightarrow \mathbb{C}$ is said to be a Jordan arc if and only if it is both continuous and one-to-one. Throughout this paper we shall identify a Jordan arc $\gamma$ with its trace $\Gamma:=\gamma([0,1])$.

In order to minimize technical details we do much of our work on a particular "annular" region which has rectilinear boundary. For the rest of the paper let $E=\{z=x+i y: 1<\max \{|x|,|y|\}<2\}, S=\{z=x+i y: \max \{|x|,|y|\}=1\}$, and $T=\{z=x+i y: \max \{|x|,|y|\}=2\}$. Let us say that a Jordan arc $\Gamma:=$

Received by the editors August 14, 1989 and, in revised form, January 15, 1991.

1980 Mathematics Subject Classification (1985 Revision). Primary 30E10, 30D55; Secondary $30 \mathrm{C} 85,47 \mathrm{~B} 20$. 
$\gamma([0,1])$ connects $S$ to $T$ if $\gamma(0) \in S, \gamma(t) \in E$ for $0<t<1$, and $\gamma(1) \in T$. If $z$ and $\zeta$ are complex numbers, then let $[z, \zeta]=\{(1-t) z+t \zeta: 0 \leq t \leq 1\}$ (observe that $[z, \zeta]=[\zeta, z])$ be the segment that connects $z$ to $\zeta$.

2. Definition. Let $G$ be a bounded, simply connected region in $\mathbb{C}$. A Jordan arc $\gamma$ is called a cross-cut of $G$ if both $\gamma(0)$ and $\gamma(1)$ are in $\partial G$ and $\gamma((0,1)) \subseteq G$.

3. Lemma. (a) Let $D$ and $G$ be bounded, simply connected regions in $\mathbb{C}$ such that $z_{0} \in D \subseteq G$. If $B$ is a Borel subset of $(\partial D) \cap(\partial G)$, then $\omega\left(B, D, z_{0}\right) \leq$ $\omega\left(B, G, z_{0}\right)$.

(b) Let $G$ be a bounded, simply connected region in $\mathbb{C}$. If $\gamma$ is a cross-cut of $G(\Gamma=\gamma([0,1]))$, the components of $G \backslash \Gamma$ are $G_{1}$ and $G_{2}, z_{0} \in G_{1}$ and $F=\left(\partial G_{2}\right) \backslash \Gamma$, then $\omega\left(F, G, z_{0}\right) \leq \omega\left(\Gamma, G_{1}, z_{0}\right)$.

Proof (sketch). Part (a) follows from the maximum principle for harmonic functions. Part (b) is a consequence of (a) and the fact that harmonic measure is a probability measure.

4. Lemma. Suppose $0<\varepsilon<1 / 4$ and $[\xi, \eta]$ is a segment of length $2 \varepsilon$ in $\{z \in E: \operatorname{Re}(z)>0\}$. Then $\omega([\xi, \eta], E \backslash[\xi, \eta],-3 / 2) \leq-1 / \log (\varepsilon)$.

Proof. Let $D=\{z \in \mathbb{C}:|z|<4\}$ and $\Delta=\{z \in \mathbb{C}:|z-((\zeta+\eta) / 2)|<\varepsilon\}$; notice that $\bar{\Delta} \subseteq\{z \in \mathbb{C}:|z|<3\}$. Since $E \backslash[\zeta, \eta] \subseteq D \backslash[\zeta, \eta]$, it follows from Lemma 3(a) that $\omega([\zeta, \eta], E \backslash[\zeta, \eta],-3 / 2) \leq \omega([\zeta, \eta], D \backslash[\zeta, \eta],-3 / 2)$. Likewise, since $D \backslash \bar{\Delta} \subseteq D \backslash[\zeta, \eta]$ we have $\omega(\partial D, D \backslash \bar{\Delta},-3 / 2) \leq \omega(\partial D, D \backslash[\zeta, \eta],-3 / 2)$, and therefore $\omega([\zeta, \eta], D \backslash[\zeta, \eta],-3 / 2) \leq \omega(\partial \Delta, D \backslash \bar{\Delta},-3 / 2)$. Consequently, $\omega([\zeta, \eta], E \backslash[\zeta, \eta],-3 / 2) \leq \omega(\partial \Delta, D \backslash \bar{\Delta},-3 / 2)$.

Next we let $\varphi$ be a Möbius transformation that maps $D$ onto the unit disk $\mathbb{D}$ and $\Delta$ onto a disk $\Delta_{\varphi}$ with center $z=0$. Elementary calculations give us that $|\varphi(-3 / 2)| \geq 3 / 8$ and that the radius of $\Delta_{\varphi}$ is at most $\varepsilon$. So

$$
\begin{aligned}
\log (3 / 8) & \leq \log |\varphi(-3 / 2)|=\int \log |z| d \omega\left(z, \mathbb{D} \backslash \bar{\Delta}_{\varphi}, \varphi(-3 / 2)\right) \\
& =\left[\log \left(\operatorname{radius}\left(\Delta_{\varphi}\right)\right)\right] \cdot \omega\left(\partial \Delta_{\varphi}, \mathbb{D} \backslash \bar{\Delta}_{\varphi}, \varphi(-3 / 2)\right) \\
& \leq[\log (\varepsilon)] \cdot \omega\left(\partial \Delta_{\varphi}, \mathbb{D} \backslash \bar{\Delta}_{\varphi}, \varphi(-3 / 2)\right) .
\end{aligned}
$$

Therefore,

$$
\begin{aligned}
& \omega([\zeta, \eta], E \backslash[\zeta, \eta],-3 / 2) \leq \omega(\partial \Delta, D \backslash \bar{\Delta},-3 / 2) \\
& \quad=\omega\left(\partial \Delta_{\varphi}, \mathbb{D} \backslash \bar{\Delta}_{\varphi}, \varphi(-3 / 2)\right) \leq \frac{\log (3 / 8)}{\log (\varepsilon)}<\frac{-1}{\log (\varepsilon)} .
\end{aligned}
$$

5. Lemma. If $\Gamma$ is a Jordan arc that connects $S$ to $T, \omega:=\omega\left(\cdot, E \backslash \Gamma, z_{0}\right)$, and $1 / z$ can be approximated by polynomials in the $L^{2}(\omega)$ norm, then the polynomials are dense in the Hardy space $H^{2}(E \backslash \Gamma)$.

Proof. Let $\varphi$ be a conformal map from $\mathbb{D}:=\{z \in \mathbb{C}:|z|<1\}$ one-to-one and onto $E \backslash \Gamma$ such that $\varphi(0)=z_{0}$, and define $\|\cdot\|: H^{2}(E \backslash \Gamma) \rightarrow \mathbb{R}$ by

$$
\|f\|^{2}=\left|f\left(z_{0}\right)\right|^{2}+\int_{E \backslash \Gamma}\left|f^{\prime}\right|^{2}\left(1-\left|\varphi^{-1}\right|^{2}\right) d A .
$$


By Green's Theorem and a change of variables, $\|\cdot\|$ defines a norm on $H^{2}(E \backslash \Gamma)$ that is equivalent to the Hardy space $H^{2}(E \backslash \Gamma)$ norm.

Now from our hypothesis it follows that no point in $\partial(E \backslash \Gamma)$ can be an analytic bounded point evaluation for the polynomials with respect to the $L^{2}(\omega)$ norm. Since the $L^{2}(\omega)$ and $H^{2}(E \backslash \Gamma)$ norms are equivalent for the polynomials, we can conclude that no point in $\partial(E \backslash \Gamma)$ is an analytic bounded point evaluation for the polynomials with respect to the $L^{2}\left(\left(1-\left|\varphi^{-1}\right|^{2}\right) d A\right)$ norm. Therefore, by [5, Theorem 4], the polynomials are dense in $L_{a}^{2}\left(E \backslash \Gamma,\left(1-\left|\varphi^{-1}\right|^{2}\right) d A\right)$, and thus are dense in $H^{2}(E \backslash \Gamma)$ by [4, Corollary 3.4].

Let $\Gamma$ be a Jordan arc that connects $S$ to $T$. How pathological must $\Gamma$ be so that the polynomials have a chance of being dense in $H^{2}(E \backslash \Gamma)$ ? If $\varphi$ is a conformal map from the unit disk $\mathbb{D}$ one-to-one and onto $E \backslash \Gamma$ and the polynomials are dense in $H^{2}(E \backslash \Gamma)$, then by [4, Corollary 3.5] $\varphi$ must be univalent almost everywhere on $\partial \mathbb{D}$. This can be rephrased in terms of $\omega\left(\cdot, E \backslash \Gamma, z_{0}\right)$ to give us that the set of tangent points of $\Gamma$ (see [3]) has onedimensional Hausdorff measure equal to zero; but much more can be said. Indeed, if there exists one point $z$ in $\Gamma$ and a crescent $\Omega$ in $E \backslash \Gamma$, with multiple boundary point $z$, such that the bounded component of $\mathbb{C} \backslash \bar{\Omega}$ contains $\{z=x+i y: \min \{|x|,|y|\} \leq 1\}$ and the polynomials are not dense ir $H^{2}(\Omega)$, then the polynomials are not dense in $H^{2}(E \backslash \Gamma)$. A consequence of this (cf. [1]) is that if there exists one point $z$ in $\Gamma$ such that from each side of $\Gamma$ we can approach $z$ through a cone in $E \backslash \Gamma$, then the polynomials are not dense in $H^{2}(E \backslash \Gamma)$.

6. Theorem. There exists a Jordan arc $\Gamma$ that connects $S$ to $T$ such that the polynomials are dense in $H^{2}(E \backslash \Gamma)$.

Proof. By Lemma 5, it is sufficient to produce a Jordan arc $\Gamma$ that connects $S$ to $T$ such that $-3 / 2 \notin \Gamma$ and $1 / z$ can be approximated by polynomials in the $L^{2}(\omega)$ norm; $\omega:=\omega(\cdot, E \backslash \Gamma,-3 / 2)$. A reasonable strategy for producing this $\Gamma$ is to find a sequence of polynomials $\left\{p_{n}\right\}$ and a sequence of polygonal Jordan arcs $\left\{\Gamma_{n}\right\}$ such that

(a) for all $n, \Gamma_{n}$ connects $S$ to $T, \operatorname{Re}(z)>0$ for all $z$ in $\Gamma_{n}$, and $\left\{\Gamma_{n}\right\}$ converges uniformly to a Jordan arc $\Gamma$ that connects $S$ to $T$;

(b) $\int\left|1 / z-p_{k}\right|^{2} d \omega_{n}<1 / k$ whenever $1 \leq k \leq n$, where $\omega_{n}:=\omega_{n}\left(\cdot, E \backslash \Gamma_{n},-3 / 2\right)$.

In fact, for convenience of proof, we shall choose $\Gamma_{n}$ so that its angle of incidence with both $S$ and $T$ is $\pi / 2$ and that the angle formed by $\Gamma_{n}$ at any of its vertices is at least $\pi / 3$. The limiting arc $\Gamma$ is the one we are after.

Let $W_{1}=\{z \in E: \operatorname{dist}(z,[1,2])<1 / 8\}$. By Runge's Theorem, there is a polynomial $p_{1}$ such that

$$
\left\|\left(1 / z-p_{1}\right)^{2}\right\|_{E \backslash W_{1}}:=\sup \left\{\left|1 / z-p_{1}(z)\right|^{2}: z \in E \backslash W_{1}\right\}<1 / 2 .
$$

Now we construct $\Gamma_{1}$. Let $\Omega_{1}=\{1-i / 4,5 / 4+i / 4,3 / 2-i / 4,7 / 4+i / 4,2-$ $i / 4\}$; obviously $5=$ cardinality of $\Omega_{1}:=\left|\Omega_{1}\right|$. Choose $0<\varepsilon_{1}<1 / 16$ small 


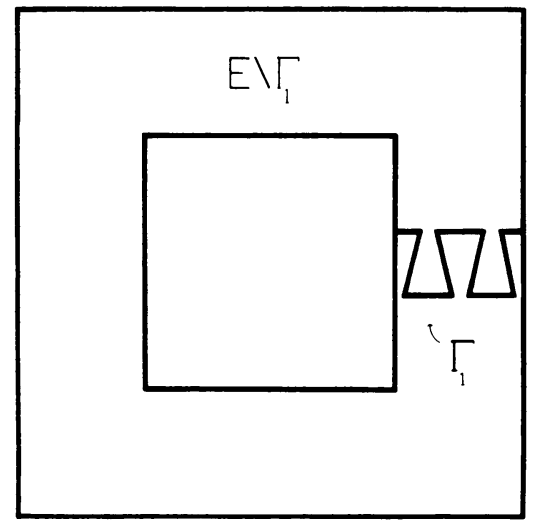

Figure 1

enough so that

$$
5\left(\frac{-1}{\log \left(\varepsilon_{1}\right)}\right)<1 /\left(2\left\|\left(1 / z-p_{1}\right)^{2}\right\|_{W_{1}}\right) .
$$

Let $K_{1}=([1+i / 4,2+i / 4] \cup[1-i / 4,2-i / 4]) \backslash \bigcup_{z \in \Omega_{1}} B\left(z ; \varepsilon_{1}\right)$, where $B\left(z ; \varepsilon_{1}\right)$ $:=\left\{\zeta \in \mathbb{C}:|z-\zeta|<\varepsilon_{1}\right\}$. Now $K_{1}$ is a closed set with five components: $I_{1}(1), I_{1}(3), I_{1}(5), I_{1}(7)$, and $I_{1}(9)$, each of which is a segment. The numbering scheme is as follows: $I_{1}(j) \subseteq[1+i / 4,2+i / 4]$ for $j=1,5,9$; $I_{1}(j) \subseteq[1-i / 4,2-i / 4]$ for $j=3,7 ; \operatorname{Re}(z)<\operatorname{Re}(\zeta)<\operatorname{Re}(\eta)$ whenever $z \in I_{1}(1), \zeta \in I_{1}(5)$, and $\eta \in I_{1}(9)$, and $\operatorname{Re}(z)<\operatorname{Re}(\zeta)$ whenever $z \in I_{1}(3)$ and $\zeta \in I_{1}(7)$. Connect the right endpoint of $I_{1}(1)$ to the left endpoint of $I_{1}(3)$, the right endpoint of $I_{1}(3)$ to the left endpoint of $I_{1}(5)$, the right endpoint of $I_{1}(5)$ to the left endpoint of $I_{1}(7)$, and the right endpoint of $I_{1}(7)$ to the left endpoint or $I_{1}(9)$, with segments $I_{1}(2), I_{1}(4), I_{1}(6)$, and $I_{1}(8)$, respectively. Let $\Gamma_{1}=\bigcup_{j=1}^{9} I_{1}(j)$ (see Figure 1). Notice that $\Gamma_{1}$ is a polygonal Jordan arc that connects $S$ to $T$, the angle of incidence of $\Gamma_{1}$ with both $S$ and $T$ is $\pi / 2$, and the angle formed by $\Gamma_{1}$ at any of its vertices is at least $\pi / 3$.

Now $\bar{W}_{1}$ is only accessible in $E \backslash \Gamma_{1}$ from $z=-3 / 2$ through five "gaps" in $\Gamma_{1}$, each of size at most $2 \varepsilon_{1}$. Consequently, by (6.2), Lemma 4, and Lemma 3(b), $\omega_{1}\left(\bar{W}_{1}\right)<1 /\left(2\left\|\left(1 / z-p_{1}\right)^{2}\right\|_{W_{1}}\right) ; \omega_{1}:=\omega_{1}\left(\cdot, E \backslash \Gamma_{1},-3 / 2\right)$. Therefore, since $\left\|\left(1 / z-p_{1}\right)^{2}\right\|_{E \backslash W_{1}}<1 / 2$, we have that

$$
\int\left|1 / z-p_{1}\right|^{2} d \omega_{1}<1 .
$$

For $n \geq 2, \Gamma_{n}$ is constructed inductively so that "over" each segment of $\Gamma_{n-1}, \Gamma_{n}$ looks like $\Gamma_{1}$. In order to construct $\Gamma_{n}$, certain other items need to be defined inductively. For $n \geq 2$, let

$$
W_{n}=\left\{z \in E: \operatorname{dist}\left(z, \Gamma_{n-1}\right)<\varepsilon_{n-1} / 16\right\}
$$

and let

$$
W_{n}^{\prime}=\left\{z \in E: \operatorname{dist}\left(z, \Gamma_{n-1}\right)<\varepsilon_{n-1} / 8\right\}
$$


By Runge's Theorem there is a polynomial $p_{n}$ such that $\left\|\left(1 / z-p_{n}\right)^{2}\right\|_{E \backslash W_{n}}<$ $1 /(2 n)$. The substance of the inductive step is found in the construction of $\Gamma_{2}$ and so, for the most part, we focus our attention there.

Recall that $\Gamma_{1}=\bigcup_{j=1}^{9} I_{1}(j)$. For $1 \leq j \leq 9$, let $I_{1}^{*}(j)$ be the straight line that contains $I_{1}(j)$, and let $D_{2}(j)=\left\{z \in \mathbb{C}: \operatorname{dist}\left(z, I^{*}(j)\right)<\varepsilon_{1} / 8\right\}$. For $j=2,4,6,8$, let $V_{2}(j, j+1)=D_{2}(J) \cap D_{2}(j+1)$ and $V_{2}(j, j-1)=$ $D_{2}(j) \cap D_{2}(j-1)$. Let

$$
W_{2}^{\prime \prime}=W_{2}^{\prime} \cup\left\{\bigcup_{j=1}^{4}\left(V_{2}(2 j, 2 j+1) \cup V_{2}(2 j, 2 j-1)\right)\right\} .
$$

Notice that, unlike $\partial W_{2}^{\prime}, \partial W_{2}^{\prime \prime}$ is a polygon. Moreover, since the angle formed by $\Gamma_{1}$ at any of its vertices is at least $\pi / 3$, it follows that $\operatorname{dist}\left(z, \Gamma_{1}\right)<\varepsilon_{1} / 4$ whenever $z \in W_{2}^{\prime \prime}$. We shall construct $\Gamma_{2}$ using $\operatorname{cl}\left\{E \cap\left(\partial W_{2}^{\prime \prime}\right)\right\}$.

Now $\partial V_{2}(j, j+1)$ [resp., $\partial V_{2}(j, j-1)$ ] is a parallelogram $(j=2,4,6,8)$. Let $a_{2}(j, j+1)$ [resp., $a_{2}(j, j-1)$ ] be the unique vertex of $\partial V_{2}(j, j+1)$ [resp., $\partial V_{2}(j, j-1)$ ] which is in $c o\left(I_{1}(j) \cup I_{1}(j+1)\right):=$ closed convex hull of $\left(I_{1}(j) \cup I_{1}(j+1)\right)$ [resp., $c o\left(I_{1}(j) \cup I_{1}(j-1)\right)$ ]. Let $b_{2}(j, j+1)$ [resp., $b_{2}(j, j-1)$ ] be the unique point in $\partial D_{2}(j+1)$ [resp., $\partial D_{2}(j-1)$ ] such that the segment $\left[a_{2}(j, j+1), b_{2}(j, j+1)\right]$ [resp., $\left[a_{2}(j, j-1), b_{2}(j, j-1)\right]$ is perpendicular to $I_{1}^{*}(j+1)$ [resp., $I_{1}^{*}(j-1)$ ], and let $c_{2}(j, j+1)$ [resp., $c_{2}(j, j-1)$ ] be the unique point in $\partial D_{2}(j)$ such that the segment $\left[a_{2}(j, j+1), c_{2}(j, j+1)\right]$ [resp., $\left.\left[a_{2}(j, j-1), c_{2}(j, j-1)\right]\right]$ is perpendicular to $I_{1}^{*}(j)$. Let $a_{2}(0,1)$ [resp., $\left.a_{2}(10,9)\right]$ be the intersection of the component of $\operatorname{cl}\left\{E \cap\left(\partial W_{2}^{\prime \prime}\right)\right\}$ that contains $a_{2}(2,1)$ with $S$ [resp., $T$ ], and let $b_{2}(0,1)$ [resp., $b_{2}(10,9)$ ] be the intersection of the component of $\operatorname{cl}\left\{E \cap\left(\partial W_{2}^{\prime \prime}\right)\right\}$ that contains $b_{2}(2,1)$ with $S$ [resp., T]. For $1 \leq j \leq 9$, if $j$ is odd, then let $R_{2}(j)$ be the rectangle with vertices $a_{2}(j+1, j), a_{2}(j-1, j), b_{2}(j+1, j)$, and $b_{2}(j-1, j)$; and if $j$ is even, then let $R_{2}(j)$ be the rectangle with vertices $a_{2}(j, j+1), a_{2}(j, j-1)$, $c_{2}(j, j+1)$, and $c_{2}(j, j-1)$. Call a rectangle $R_{2}(j)$ even if its $a_{2}$-vertices are diagonal, and odd otherwise. Notice that $R_{2}(j)$ is even if $j$ is odd, and odd if $j$ is even.

With straight lines that are perpendicular to $I_{2}^{*}(j)$, partition $R_{2}(j)$ into congruent subrectangles so that the number of subrectangles is even [resp., odd] if $R_{2}(j)$ is even [resp., odd], the greatest dimension of any subrectangle is $\varepsilon_{1} / 4$ (the width of $R_{2}(j)$ ) and the least dimension is no less than $\varepsilon_{1} / 8$; it is possible to partition in this way because the length of any $R_{2}(j)$ is at least twice its width-see Figure 2 on next page (labeled in part). Let $\Omega_{2}$ be the collection of points defined by:

(i) $a_{2}(0,1) \in \Omega_{2}$

(ii) $z \in \Omega_{2}$ if and only if $z$ is a vertex of some subrectangle of some $R_{2}(j)$ and the vertex diagonal to $z$ in this subrectangle is in $\Omega_{2}$. that

Now choose $0<\varepsilon_{2}<\varepsilon_{1} / 16$ (for $n \geq 2,0<\varepsilon_{n}<\varepsilon_{n-1} / 16$ ) small enough so

$$
\left|\Omega_{2}\right|\left(-1 / \log \left(\varepsilon_{2}\right)\right)<1 /\left(4\left\|\left(1 / z-p_{2}\right)^{2}\right\|_{W_{2}}\right),
$$

and let $K_{2}=\operatorname{cl}\left\{E \cap\left(\partial W_{2}^{\prime \prime}\right)\right\} \backslash \bigcup_{z \in \Omega_{2}} B\left(z ; \varepsilon_{2}\right)$. Notice that $K_{2}$ is made up of finitely many components, each of which is either a segment or a polygonal Jordan arc that is the union of two segments. In the same way that $\Gamma_{1}$ was pieced 


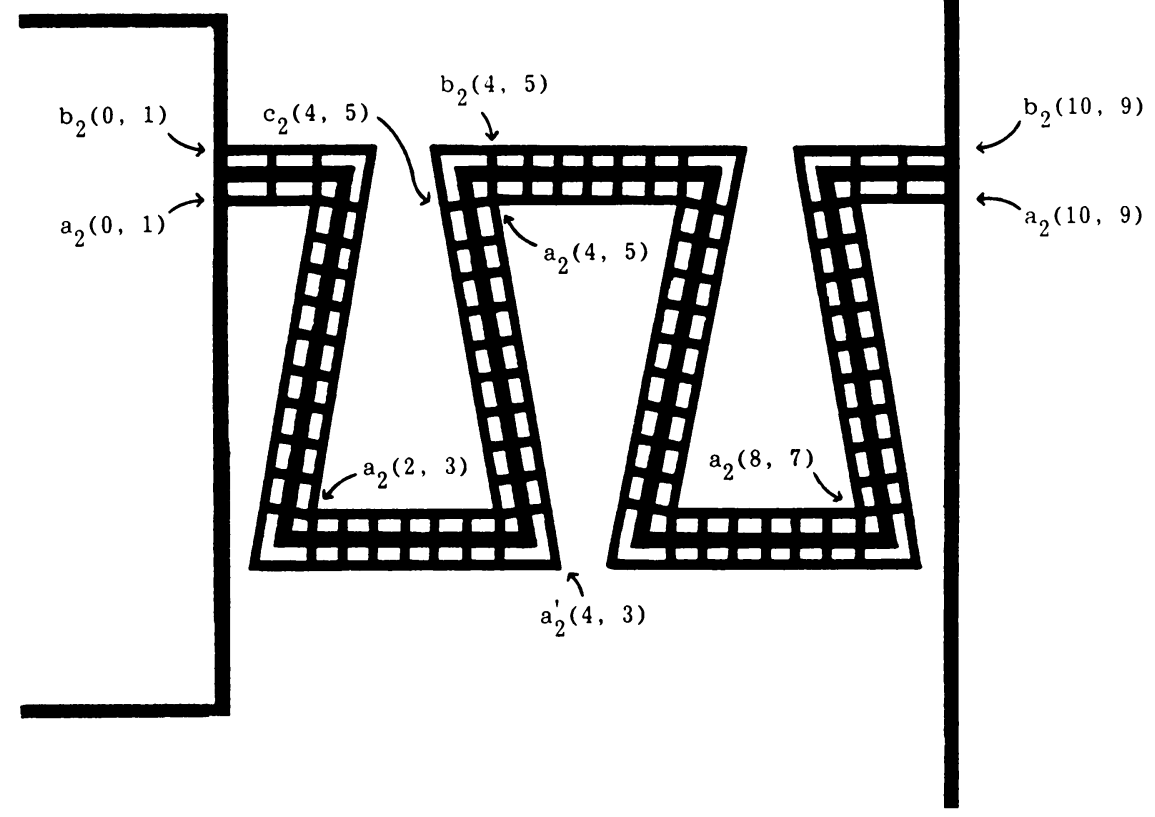

FIGURE 2

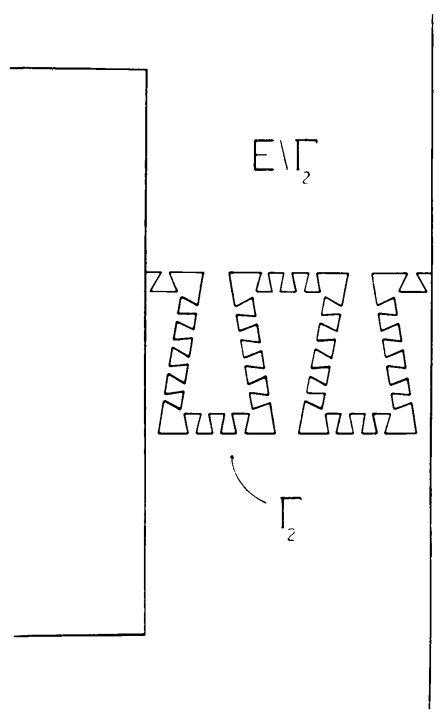

FIGURE 3

together, construct $\Gamma_{2}$ by connecting, with segments, the right endpoint of the component of $K_{2}$ which contains $b_{2}(0,1)$ to the left endpoint of the component which contains the vertex that is diagonal to $b_{2}(0,1)$ in the subrectangle of $R_{2}(1)$ which has $b_{2}(0,1)$ as a vertex, etc. (see Figure 3 ). The resulting arc $\Gamma_{2}$ is a polygonal Jordan arc whose angle of incidence with both $S$ and $T$ is 
$\pi / 2$ and whose angle at any vertex is at least $\pi / 3$. Moreover, any maximal segment of $\Gamma_{2}$ (i.e., a segment of $\Gamma_{2}$ that is properly contained in no other segment of $\left.\Gamma_{2}\right)$ has length at least $2 \varepsilon_{2}$. Now since $\left\|\left(1 / z-p_{2}\right)^{2}\right\|_{E \backslash W_{2}}<1 / 4$ and $\bar{W}_{2}$ is only accessible in $E \backslash \Gamma_{2}$ from $z=-3 / 2$ through $\left|\Omega_{2}\right|$ "gaps" in $\Gamma_{2}$ each of size at most $2 \varepsilon_{2}$, it follows from (6.3), Lemma 4, and Lemma 3(b) that

$$
\int\left|1 / z-p_{2}\right|^{2} d \omega_{2}<1 / 2
$$

where $\omega_{2}:=\omega_{2}\left(\cdot, E \backslash \Gamma_{2},-3 / 2\right)$. Also notice that by our choice of $\Gamma_{2}$, in order to access $\bar{W}_{1}$ in $E \backslash \Gamma_{2}$ from $z=-3 / 2$, one must pass through one of five gaps in $\Gamma_{2}$, each of which represents a narrowing of one of the gaps in $\Gamma_{1}$. Consequently,

$$
\int\left|1 / z-p_{1}\right|^{2} d \omega_{2}<1
$$

For $n \geq 3, p_{n}$ is chosen and $\Gamma_{n}$ is constructed in basically the same way we chose $p_{2}$ and constructed $\Gamma_{2}$.

Let us parametrize $\Gamma_{n}$. Define $\gamma_{1}:[0,1] \rightarrow \Gamma_{1}$ by $\gamma_{1}(x)$ is the point on $\Gamma_{1}$ whose distance along $\Gamma_{1}$ from $S$ is $x \cdot$ [length $\left.\left(\Gamma_{1}\right)\right]$. Now we turn to $\Gamma_{2}$. For $j=2,4,6,8$ let $a_{2}^{\prime}(j, j+1)$ [resp., $a_{2}^{\prime}(j, j-1)$ ] be the vertex of $\partial V(j, j+1)$ [resp., $\partial V(j, j-1)$ ] that is diagonal to $a_{2}(j, j+1)$ [resp., $\left.a_{2}(j, j-1)\right]$. Notice that $a_{2}^{\prime}(j, j+1)$ and $a_{2}^{\prime}(j, j-1)$ are in $\Gamma_{2}$. Now $\Gamma_{2} \backslash\left\{\bigcup_{i=1}^{4}\left\{a_{2}^{\prime}(2 i, 2 i+1), a_{2}^{\prime}(2 i, 2 i-1)\right\}\right\}$ has nine components; number them as to the order in which each is encountered when traversing $\Gamma_{2}$ from $S$ to $T$. Define a continuous one-to-one function $\beta_{2}: \Gamma_{1} \rightarrow \Gamma_{2}$ by mapping $I_{1}(j)$ (recall that $\Gamma_{1}=\bigcup_{j=1}^{9} I_{1}(j)$ ) onto the closure of the $j$ th component of $\Gamma_{2} \backslash\left\{\bigcup_{i=1}^{4}\left\{a_{2}^{\prime}(2 i, 2 i+1), a_{2}^{\prime}(2 i, 2 i-1)\right\}\right\}$ in the same way that $\gamma_{1}$ maps $[0,1]$ onto $\Gamma_{1}$. Let $\gamma_{2}=\beta_{2} \circ \gamma_{1}$. Similarly, for any $n$, define a continuous one-toone function $\beta_{n}: \Gamma_{n-1} \rightarrow \Gamma_{n}$ by mapping any maximal segment of $\Gamma_{n-1}$ (i.e., a segment of $\Gamma_{n-1}$ that is properly contained in no other segment of $\left.\Gamma_{n-1}\right)$ to the part of $\Gamma_{n}$ that "covers" the segment. Then let $\gamma_{n}=\beta_{n} \circ \gamma_{n-1}$.

Choose $\delta>0$. Now there exists $N \geq 3$ such that $\varepsilon_{n-2}<\delta$. No maximal segment of $\Gamma_{n-1}$ has length greater than $(3 / 4) \cdot \varepsilon_{N-2}$. So, by the construction of $\Gamma_{k}$ and the definition of $\gamma_{k}$, if $m, n \geq N$ and $t \in[0,1]$, then $\left|\gamma_{m}(t)-\gamma_{n}(t)\right|<$ $\delta$. Therefore, $\left\{\gamma_{n}\right\}$ is uniformly Cauchy and hence converges uniformly to a continuous function $\gamma:[0,1] \rightarrow \Gamma:=\gamma([0,1])$.

To show that $\gamma$ is one-to-one, choose $s$ and $t$ in $[0,1]$ such that $s \neq$ $t$. By the definition of $\gamma_{n}$ there exists $N$ such that $\gamma_{N}(s)$ and $\gamma_{N}(t)$ are in nonadjacent maximal segments of $\Gamma_{N}$. Reviewing the construction of $\Gamma_{N}$, we find that $\left|\gamma_{N}(s)-\gamma_{N}(t)\right| \geq 2 \varepsilon_{N}$. In fact, if $n \geq N+1$, then

$$
\left|\gamma_{n}(s)-\gamma_{n}(t)\right| \geq 2 \varepsilon_{N}-2 \cdot \sum_{k=N+1}^{n} \frac{\varepsilon_{n}}{4^{k-N}}>\varepsilon_{N} .
$$

Hence, $\left|\gamma_{n}(s)-\gamma_{n}(t)\right| \nrightarrow 0$ as $n \rightarrow \infty$, and so $\gamma(s) \neq \gamma(t)$. Therefore $\gamma$ is one-to-one, and $\Gamma$ is a Jordan arc.

We now have a sequence of polynomials $\left\{p_{n}\right\}$ and a sequence of polygonal Jordan $\operatorname{arcs}\left\{\Gamma_{n}\right\}$ which satisfy $(6.1)(\mathrm{a})$ and (b). Let $\omega:=\omega(\cdot, E \backslash \Gamma,-3 / 2)$. 
Since $\Gamma_{n}$ converges uniformly to $\Gamma$, it follows that, for fixed $k$,

$$
\int\left|1 / z-p_{k}\right|^{2} d \omega_{n} \rightarrow \int\left|1 / z-p_{k}\right|^{2} d \omega
$$

as $n \rightarrow \infty ; \omega_{n}:=\omega_{n}\left(\cdot, E \backslash \Gamma_{n},-3 / 2\right)$. Therefore, because $\int\left|1 / z-p_{k}\right|^{2} d \omega_{n}<$ $1 / k$ whenever $1 \leq k \leq n$, we have that

$$
\int\left|1 / z-p_{k}\right|^{2} d \omega \leq 1 / k \rightarrow 0,
$$

as $k \rightarrow \infty$. by Lemma 5 , the proof is now complete.

7. Theorem. There exists a Jordan $\Gamma:=\gamma([0,1])$, where $\gamma(0)=0, \gamma(t) \in \mathbb{D}:=$ $\{z \in \mathbb{C}:|z|<1\}$ for $0<t<1$, and $\gamma(1)=1$, such that the polynomials are dense in $H^{2}(\mathbb{D} \backslash \Gamma)$.

Proof (sketch). In a way similar to the proof of Theorem 4, we produce a sequence of Jordan arcs $\left\{\Gamma_{n}:=\gamma_{n}([0,1])\right\} \quad\left(\Gamma_{n}\right.$ having the same geometry as in the proof of Theorem 4), where $\gamma_{n}([0,1]) \subseteq\{z \in \mathbb{D}: \operatorname{Re}(z)>0\}$ and $\left|\gamma_{n}(1)\right|=1$ for all $n$, a sequence of points $\left\{t_{n}\right\}$, where $0<t_{n}<1$ and $\left|\gamma_{n}\left(t_{n}\right)-\gamma_{n}(0)\right| \rightarrow 0$ as $n \rightarrow \infty$, and a sequence of polynomials $\left\{p_{n}\right\}$ such that:

(a) $\Gamma_{n}$ converges uniformly to a Jordan arc $\Gamma:=\gamma([0,1])$, where $\gamma(0)=$ $0, \gamma(t) \in \mathbb{D}$ for $0<t<1$, and $\gamma(1)=1$;

(b) $\left|p_{n}\left(\gamma_{n}\left(t_{n}\right)\right)\right| \geq 1$ for all $n$, and $\int\left|p_{k}\right|^{2} d \omega_{n}<1 / k$ whenever $1 \leq k \leq n$, where $\omega_{n}:=\omega_{n}\left(\cdot, \mathbb{D} \backslash \Gamma_{n},-1 / 2\right)$.

The limiting arc $\Gamma:=\gamma([0,1])$ will then have the property that, for all $n$, $\int\left|p_{n}\right|^{2} d \omega \leq 1 / n(\omega:=\omega(\cdot, \mathbb{D} \backslash \Gamma,-1 / 2))$ and yet $\left|p_{n}\left(\gamma_{n}\left(t_{n}\right)\right)\right| \geq 1$, where $\gamma_{n}\left(t_{n}\right) \rightarrow \gamma(0)$ as $n \rightarrow \infty$. So, $\gamma(0)$ is not an analytic bounded point evaluation for the polynomials with respect to the $H^{2}(\mathbb{D} \backslash \Gamma)$ norm, and hence nor is any point in $\partial(\mathbb{D} \backslash \Gamma)$. Following an argument similar to the proof of Lemma 3 , we get that the polynomials are dense in $H^{2}(\mathbb{D} \backslash \Gamma)$.

8. Remark. Theorems 4 and 5 provide us with new examples of analytic Toeplitz operators $T_{\varphi}$, where $\varphi$ is a Riemann map from the unit disk $\mathbb{D}$ onto $E \backslash \Gamma$ (of Theorem 4) or onto $\mathbb{D} \backslash \Gamma$ (of Theorem 5), such that $T_{\varphi}$ is cyclic (with cyclic vector 1) and yet $\varphi$ is not a weak-star generator of $H^{\infty}$ (cf. [8]).

There is unfinished business here, and yet very little of it is easily approachable.

9. Problem. Find a condition on $\Gamma$ which is both necessary and sufficient for density of the polynomials in $H^{2}(\mathbb{D} \backslash \Gamma)$, where $\Gamma:=\gamma([0,1])$ is a Jordan arc such that $\gamma([0,1)) \subseteq \mathbb{D}$ and $\gamma(1)=1$.

10. Question. Does there exist a Jordan arc $\Gamma$, with endpoints 0 and 1 , such that the polynomials are dense in $L_{a}^{2}(\mathbb{D} \backslash \Gamma, d A)$ ?

\section{ACKNOWLEDGMENT}

The author is grateful to Daniel Luecking for pointing out some useful references and to the referee for helpful suggestions. 


\section{REFERENCES}

1. J. Akeroyd, Polynomial approximation in the mean with respect to harmonic measure on crescents, Trans. Amer. Math. Soc. 303 (1987), 193-199.

2. __ Point evaluations and polynomial approximation in the mean with respect to harmonic measure, Proc. Amer. Math. Soc. 105 (1989), 575-581.

3. C. J. Bishop, L. Carleson, J. B. Garnett, and P. W. Jones, Harmonic measures supported on curves, Pacific J. Math. 138 (1989), 233-236.

4. P. S. Bourdon, Density of the polynomials in Bergman spaces, Pacific J. Math. 130 (1987), 215-221.

5. J. Brennan, Point evaluations, invariant subspaces and approximation in the mean polynomials, J. Funct. Anal. 34 (1979), 407-420.

6. P. L. Duren, Theory of $H^{p}$-spaces, Academic Press, New York, 1970.

7. T. W. Gamelin, Uniform algebras, 2nd ed., Chelsea, New York, 1984.

8. R. C. Roan, Composition operators on $H^{p}$ with dense range, Indiana Univ. Math. J. 27 (1978), 159-162.

Department of Mathematics, University of ARKansas at Fayetteville, Fayetteville, ARKANSAS 72701 